\title{
Kernos
}

Revue internationale et pluridisciplinaire de religion grecque antique

$25 \mid 2012$

Varia

\section{Gabrielle FRIJA, Les Prêtres des empereurs. Le culte impérial civique dans la province romaine d'Asie}

\section{Domitilla Campanille}

\section{OpenEdition \\ Journals}

\section{Edizione digitale}

URL: http://journals.openedition.org/kernos/2080

DOI: $10.4000 /$ kernos.2080

ISSN: 2034-7871

\section{Editore}

Centre international d'étude de la religion grecque antique

\section{Edizione cartacea}

Data di pubblicazione: 26 octobre 2012

Paginazione: $374-377$

ISSN: 0776-3824

Notizia bibliografica digitale

Domitilla Campanille, « Gabrielle friJA, Les Prêtres des empereurs. Le culte impérial civique dans la province romaine d'Asie », Kernos [En ligne], 25 | 2012, mis en ligne le 01 octobre 2012, consulté le 21 septembre 2020. URL : http://journals.openedition.org/kernos/2080 ; DOI : https://doi.org/10.4000/ kernos. 2080

Questo documento è stato generato automaticamente il 21 septembre 2020.

Kernos 


\title{
Gabrielle FRIJA, Les Prêtres des empereurs. Le culte impérial civique dans la province romaine d'Asie
}

\author{
Domitilla Campanille
}

\section{NOTIZIA}

Gabrielle FRIJA, Les Prêtres des empereurs. Le culte impérial civique dans la province romaine d'Asie, Rennes, Presses universitaires de Rennes, 2012. 1 vol. 15,5 × $24 \mathrm{~cm}, 323 \mathrm{p}$.

(Collection " Histoire », série " Histoire ancienne »). ISBN : 978-2-7535-1738-7.

1 Nel 29 a.C. nella provincia d'Asia e in quella di Bitinia, regioni fra le più ricche, urbanizzate ed ellenizzate della penisola anatolica allora soggetta al dominio romano, venne istituito il culto provinciale del vivente princeps. Cassio Dione (LI, 20, 6, cfr. Tacito, Annales IV, 37) afferma che in quell'anno Ottaviano permise ai Romani residenti in Asia e in Bitinia di erigere un tempio dedicato a Roma e a Giulio Cesare nelle città di Efeso e di Nicea. Ai peregrini, ovvero ai Greci d'Asia e di Bitinia Ottaviano concesse invece di innalzare un tempio dedicato a sé e alla dea Roma nelle città di Pergamo e Nicomedia. La data segna allora l'inizio di quel fenomeno denominato culto imperiale. A partire da questo stesso momento le città d'Asia cominciarono a celebrare singolarmente un culto in onore dell'imperatore.

Il volume di Gabrielle Frija (G.F.)è dedicato appunto allo studio dei sommi sacerdoti e sacerdotesse del culto imperiale cittadino. Come spiega la studiosa all'inizio della ricerca (p. 13): “À partir d’une prosopographie des prêtres des empereurs dans les cités de la province d'Asie, il s'agira de comprendre comment le culte des empereurs s'est inséré dans les institutions politiques et religieuses civiques d'une part, ce qu'il a représenté pour les milieux dirigeants des cités d'autre part". La ricerca è strutturata tematicamente: a un'introduzione cronologica e geografica (Chronologie et facteurs de diffusion des prêtrises des empereurs, p. 21-69) seguono tre capitoli, uno su Les prêtrises des 
empereurs dans la vie institutionelle des cités (p.71-111), l'altro su Les sacerdoces des empereurs dans la religion de la cité (p. 113-167), mentre l'ultimo delinea un Portrait social des prêtres du culte impérial civique (p. 169-216). Una Conclusion générale (p. 217-219) chiude la ricerca che è completata dalla Liste des prêtres du culte impérial civique (p. 223-273), da 4 carte di distribuzione, dalla Bibliographie (p. 279-295), dall' Index des sources (p. 297-307), da un Index des noms de personne (p. 308-315) e un Index géographique (p. 315-319).

3 Il culto dell'imperatore è presente in tutto l'impero, ma l'ambito geografico e cronologico privilegiato è scelto con giudizio; il territorio dell'antica provincia d'Asia, infatti, è stato e continua a essere tra le regioni dell'impero romano una fra le più generose nel restituire una documentazione che permette di studiare in forma non episodica molti aspetti della vita politica, culturale, sociale ed economica di quei luoghi. Nel caso specifico, poi, è del tutto giustificato studiare un fenomeno diffusosi in tutto l'impero a cominciare dal luogo ove il fenomeno ha avuto origine. Una tale opzione permette di studiarne la genesi, il significato originario, le prime fasi, le varianti nella titolatura iniziale dei sommi sacerdoti, i rapporti con altre forme cultuali verso individui viventi, entità e istituzioni come la dea Roma o il Senato (p. 24-36). In questo modo, poi, può emergere compiutamente il contributo significativo che singoli notabili delle città d'Asia hanno apportato alla fondazione del culto locale dell'imperatore all'indomani della vittoria di Azio (p. 37): "Les cités pionnières dans la fondation du culte impérial ont donc en commun leur lien privilegié avec le pouvoir romain par l'intermédiaire de grands notables d'envergure provinciale. Mais la diffusion du culte civique est rapide et relativement large, bien qu'elle concerne surtout la partie occidentale de la province."

4 È indispensabile aggiungere subito che l'A. ha reso disponibile sin dal mese di novembre del 2009 all'indirizzo <www.pretres-civiques.org> la prosopografia dei sommi sacerdoti del culto imperiale sotto forma di una ricca banca dati regolarmente aggiornata. Si tratta di uno strumento scientifico prezioso che consente un numero impressionante di ricerche e accompagna in modo estremamente funzionale questo volume: nel volume è presente la lista dei sacerdoti e delle sacerdotesse con le informazioni essenziali su ciascuno, le vere e proprie schede prosopografiche provviste di apparato erudito e documentazione sono ricuperabili nel sito appena citato. Siamo di fronte a un'interazione che a mio vedere potrà costituire un modello per lavori futuri.

5 Le ricerche degli storici si sono finora concentrate su problemi relativi alle figure dei sommi sacerdoti provinciali, di quei personaggi cioè che erano stati sommi sacerdoti del culto imperiale nelle città neocore, ovvero di quelle città che - grazie a un processo di selezione e a una complessa procedura che prevedeva il consenso del Senato e dell'imperatore - erano riuscite a conquistare l'ambito privilegio di essere sede di un tempio dedicato al culto imperiale. L'indagine di G.F. è invece principalmente diretta ai personaggi che hanno amministrato localmente il culto. Questa scelta porta ad analizzare in modo puntuale il funzionamento del culto all'interno di contesti religiosi e culturali specifici. Il quadro privilegiato risulta essere quindi quello delle comunità cittadine e particolare attenzione è dedicata al momento iniziale dell'instaurazione del culto civico e ai motivi specifici che ne hanno determinato la nascita nelle città interessate. Anche i rapporti tra il culto locale, quello provinciale e altre espressioni cultuali sono trattati con attenzione; il ruolo e l'influenza dell'assemblea provinciale 
d'Asia è oggetto di una trattazione adeguata e soddisfacente e sono presi in considerazione anche altri koinà attivi all'interno della provincia.

6 È evidente il valore di questo studio: permette di conoscere e approfondire un fenomeno profondamente inserito nella vita cittadina e non soggetto a selezioni e controlli esterni, a differenza di quanto poteva verificarsi per i massimi esponenti del culto imperiale provinciale. Consente, poi, di penetrare nel più ampio fenomeno della romanizzazione. Attraverso una ricerca specifica si può affrontare questo problema capitale evitando generalizzazioni e modalità puramente teoriche. Allo stesso tempo è possibile indagare il fenomeno, meno studiato ma assai rilevante, della progressiva ellenizzazione di esponenti italici insediatisi stabilmente nella provincia e dei loro discendenti. G.F. ha qui l'occasione di indagare il comportamento delle élites dirigenti cittadine nei confronti del culto imperiale. Attraverso una prosopografia dei sacerdoti è possibile individuare una fitta rete di relazioni utile anche a illuminare, per esempio, aspetti particolari delle dinamiche sociali a livello locale.

7 Bisogna sottolineare che, mentre gli studiosi disponevano da tempo di elenchi di asiarchi, sommi sacerdoti e sacerdotesse d'Asia (basti ricordare tra le varie raccolte quelle di D. Magie, J. Deininger o M. Rossner, ma pionieristici elenchi si trovano anche nel CIG), raccolte certo da aggiornare, correggere o restaurare ma sempre ottime basi da cui partire, la situazione per i sacerdoti e le sacerdotesse del culto locale era ben diversa. G.F. ha dovuto intraprendere un cospicuo lavoro di raccolta preliminare. A personaggi minori o documentati solo da esigui frammenti epigrafici si affiancavano, inoltre, individui notissimi ma dal profilo biografico di assai complessa ricostruzione, individui come G. Stertinio Senofonte di Cos [8], Potamone di Mitilene [28], G. Giulio Epicrate di Mileto [162], Antonia Tryphaena [86]1․ La costituzione di un catalogo prosopografico ove sono censiti 461 personaggi attestati in 80 città ha quindi reso possibile un'analisi complessiva in grado di portare all'ampia sintesi nella quale si sostanzia la ricerca. I personaggi sono disposti secondo un ordinamento geografico e, all'interno di questo, cronologico. La classificazione delle città segue quella del Bulletin Épigraphique. Come si è detto, la difficile e sagace opera di sintesi ha potuto avere luogo solo dopo un lungo e faticoso lavoro di raccolta dei dati e l'utilizzo e la valorizzazione di una quantità davvero notevole di documentazione epigrafica e numismatica. Lo studio, infatti, parte dalla raccolta dei dati ma riesce a porre domande decisive sul ruolo di questi sacerdoti. Attraverso una serrata analisi di materiali difficili e poco gratificanti si riescono a trovare risposte significative a varie questioni, tra le quali vorrei soffermarmi su quella - apparentemente secondaria, in realtà cruciale - del significato politico e diplomatico del culto imperiale (p. 13-18 e 99 sq.).

Concordo infatti, con quanto argomenta F.G.: sarebbe inadeguato considerare il culto espresso dai provinciali d'Asia verso l'imperatore soltanto come un compromesso particolarmente confacente alle parti, alla mentalità, alle aspirazioni e al desiderio di ascesa sociale delle élites provinciali, come al bisogno di ordine, di consenso, di pace, di fedeltà dei principes. Non si può neppure pensare che il culto tributato all'imperatore potesse essere solo una raffinata modalità espressiva elaborata dalla diplomazia greca. È chiaro che questa componente poteva essere presente, tra le varie, ma la nostra distanza culturale di fronte a simili fatti religiosi non deve impedirci di capire che strumento di integrazione e sentimento religioso non sono fenomeni reciprocamente esclusivi. La natura e i modi di espressione dei sentimenti religiosi variano da un'epoca all'altra, a seconda delle modalità di organizzazione, del tipo di cultura e 
dell'immaginario di ogni società. Se consideriamo i toni e il contenuto dei documenti prodotti dal koinòn d'Asia in occasione dell'introduzione nel 9 a.C. del nuovo calendario $^{2}$, toni e contenuto ricchi di accenti che esaltano, in una prospettiva quasi messianica, il benessere raggiunto grazie alla prosperità nuova e alla giustizia assicurata dall'imperatore, possiamo percepire la limitatezza di una concezione puramente utilitaristica o diplomatica del fenomeno del culto dell'imperatore in $\mathrm{Asia}^{3}$.

L'analisi di G.F. apporta elementi a mio vedere decisivi in questa direzione (p. 99): "Le sens politique et diplomatique du culte impérial a longtemps été le seul aspect mis en avant dans l'historiographie. Le "bon sens ", pour un esprit contemporain, conduit à interpréter la mise en place d'un culte de souverain comme un geste de loyauté, voire de flatterie politique. Il s'agit ici d'étudier la validité de cette interprétation en s'interrogeant sur le rôle des prêtres des empereurs comme représentants des cités visà-vis de l'extérieur. En effet, si la mise en place d'un culte de l'empereur est une façon de manifester sa loyauté à l'égard du pouvoir romain, on peut s'attendre à ce que le prêtres du culte impérial jouent un rôle spécifique dans la vie diplomatique de l'Orient romain." In effetti le dinamiche erano diverse e grazie all'analisi delle fonti numismatiche ed epigrafiche G.F. può smentire "le "bon sens »" e mostrare da un lato (p. 99-105) che, salvo casi particolari ben determinati, le città non utilizzavano i sacerdoti quando desideravano promuovere la propria identità e la propria immagine, dall'altro (p.105-111) che i sacerdoti non assumevano usualmente la funzione di ambasciatori né rivestivano un rango o un ruolo giuridico preciso. Era dunque la dimensione religiosa e sociale quella maggiormente soddisfatta dal culto imperiale cittadino, non quella politica o diplomatica.

La ricerca progredisce ulteriormente, G.F. riesce infatti a individuare gli ambiti privilegiati di attività per i sacerdoti, ambiti pertinenti a sacrifici e preghiere, giochi gladiatori, banchetti, (p. 145-167), a indagare la consistenza sociale dei sacerdoti e delle loro famiglie, il loro statuto giuridico, il relativo possesso della cittadinanza romana attraverso un'accurata indagine dei gentilizi, i titoli onorifici conferiti loro dalle rispettive comunità (p. 173-215)4.

11 Vengono poi individuati i luoghi di origine dei sacerdoti, si rilevano assenze significative che rispecchiano quelle relative ai sacerdoti provinciali e si nota lo scarso coinvolgimento delle isole dell'Egeo nel culto imperiale, sia cittadino sia provinciale. Un tale fenomeno invita alla riflessione. Se non intendiamo spiegarlo semplicemente con uno scarso popolamento stabile di quei territori, con la casualità dei rinvenimenti documentari, con la realtà urbanistica locale, con l'interesse da parte di studiosi più orientati a ricuperare materiali pertinenti alle epoche arcaiche e classiche, ci sarebbe da domandarsi se queste isole in età romana non fossero in effetti più isolate di quanto solitamente le riteniamo. Di contro, particolarmente numerosi risultano essere $\mathrm{i}$ sacerdoti attestati nelle città libere e il numero di 66 personaggi documentati ad Afrodisiade è piuttosto impressionante.

Rimane solo, in conclusione, da esprimere la soddisfazione di poter disporre di questo studio eccellente. 


\section{NOTE}

1. Sulla quale vd. J. THORNTON, «Una città e due regine. Eleutheria e lotta politica a Cizico fra gli Attalidi e i Giulio Claudi», Mediterraneo Antico 2/2 (1999), p. 497-538

2. Quindi vent'anni dopo l'istituzione del culto di Ottaviano, vd. l'edizione di U. LAFFI, «Le iscrizioni relative all'introduzione nel 9 a.C. del nuovo calendario della provincia d'Asia», SCO 16 (1967), p. 5-98

3. Su ciò vd. D. CAMPANILE, «Ancora sul culto imperiale in Asia», Mediterranei Antico 4/2 [2001], p. 473-488; sempre da meditare S. MAZZARINo, L'impero romano, 2, Roma/Bari, 1991 ${ }^{4}$, p. 154 sq.

4. Sui titoli vd. anche D. ERKELENZ, «Keine Konkurrenz zum Kaiser - Zur Verleihung der Titel

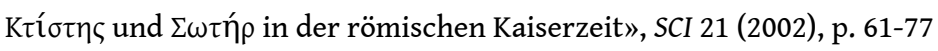

\section{AUTORI}

DOMITILLA CAMPANILLE

Università di Pisa 